\title{
School Children's Physical Activity, Motor Competence, and Corresponding Self-Perception: A Longitudinal Analysis of Reciprocal Relationships
}

\author{
Jeffrey Sallen, Christian Andrä, Sebastian Ludyga, Manuel Mücke, and Christian Herrmann
}

\begin{abstract}
Background: The relationship between engagement in physical activity and the development of motor competence (MC) is considered to be reciprocal and dynamic throughout childhood and adolescence. The 10-month follow-up study aimed to explore this reciprocal relationship and investigated whether the relationship is mediated by the corresponding self-perception of MC (PMC). Methods: A total of 51 children aged between 10 and 11 years $(M=10.27$ [0.45]) participated in the study (52.9\% boys, $47.1 \%$ girls). As an indicator for physical activity, the average vigorous physical activity (VPA) per day was measured by ActiGraph accelerometers. Two aspects of MC and PMC were recorded: self-movement and object movement. Saturated pathway models in a cross-lagged panel design with 2 measurement points were analyzed. Results: Reciprocal and direct relationships between VPA and MC object movement respectively MC self-movement were not found in longitudinal analyses with PMC as a mediator. Indirect effects of MC at $\mathrm{t} 1$ on VPA at $\mathrm{t} 2$ via PMC were identified (self-movement: $\beta=0.13,95 \%$ confidence interval, 0.04 to 0.26 ; object movement: $\beta=0.14,95 \%$ confidence interval, 0.01 to 0.49 ). Conclusion: The results highlight the importance of MC and PMC in promoting children's VPA. However, VPA does not drive the development of MC.
\end{abstract}

Keywords: MOBAK, SEMOK, fundamental movement skills, motor proficiency

Physical activity (PA) is considered to be important for health and well-being. ${ }^{1,2}$ The promotion of PA is therefore a priority for public health agencies, as well as one of the central objectives of interventions in the field of disease prevention and health promotion. $^{3,4}$ While interventions can improve PA, it remains to be clarified which correlates should be targeted to ensure that interventions for children and adolescents are optimized. ${ }^{5}$

Theoretical frameworks and empirical studies regarding children's motor development have focused on the association between motor competence (MC) and PA. ${ }^{6,7}$ Stodden et $\mathrm{al}^{8}$ assumed that the development of $\mathrm{MC}$ is a primary underlying causal mechanism that is partially responsible for commitment and persistence in PA. They described the relationship between PA and MC as reciprocal and developmentally dynamic throughout an individual's lifespan. It is hypothesized that the relationship among variable levels of MC and PA is weak in early childhood and will strengthen with the

(C) 2020 The Authors. Published by Human Kinetics, Inc. This is an Open Access article distributed under the terms of the Creative Commons Attribution 4.0 International License, CC BY 4.0, which permits unrestricted noncommercial and commercial use, distribution, and reproduction in any medium, provided the original work is properly cited, the new use includes a link to the license, and any changes are indicated. See http://creativecommons.org/licenses/by-nc/4.0. This license does not cover any third-party material that may appear with permission in the article.

Sallen is with the Department for Sport and Health Sciences, Faculty of Human Sciences, University of Potsdam, Potsdam, Germany. Andrä is with the Center for Teacher Education and School Research, University of Leipzig, Leipzig, Germany; and the Institute of Sports Psychology and Physical Education, Faculty of Sports Science, University of Leipzig, Leipzig, Germany. Ludyga, Mücke, and Herrmann are with the Department of Sport, Exercise and Health, University of Basel, Basel, Switzerland. Herrmann is also with the Zurich University of Teacher Education, Zurich, Switzerland. Sallen (sallen@uni-potsdam.de) is corresponding author. transition to late childhood. In addition, Stodden et $\mathrm{al}^{8}$ assumed that, in early childhood, PA is mainly responsible for the development of MC, whereas this relationship changes with the transition to later childhood, and MC increasingly drives PA.

In their theoretical concept, Stodden et $\mathrm{al}^{8}$ highlighted perceived $\mathrm{MC}$ (PMC) as a mediator (besides health-related fitness) that differentially influences the relationship between PA and MC over time. $\mathrm{PMC}$ refers to an individual's perception of his or her actual MC. This self-perception of MC may be understood as a latent construct based on subjective self-assessments of single instances of performing specific motor tasks (eg, catching or throwing a ball). It is a result of continually assessing one's performance in terms of meeting specific demands and combining these assessments into a general self-perception of MC. ${ }^{9}$ Estevan and Barnett ${ }^{10}$ proposed a hierarchical and multidimensional structure of a global self-concept, with PMC considered to be a subdomain of perceived sport/athletic competence, and subdivided it into different subdomains (eg, locomotion, object control). Stodden et $\mathrm{al}^{8}$ hypothesized that children can increasingly assess their actual MC more realistically with the transition from middle to late childhood. At these stages of development, actual MC drives PA not only directly but also indirectly via PMC.

Recent reviews have provided support for the theoretical concept developed by Stodden et al, ${ }^{8}$ mainly based on crosssectional studies involving children. ${ }^{7,11-15}$ Key points of these reviews are the following: (1) evidence indicates positive associations between MC, PMC, PA, and health-related physical fitness (HRF); (2) there is preliminary support for PMC as a mediator; (3) the results on the relationships between the variables of interest do appear to depend on the mode of their operationalization and the age or developmental stage of the study participants; (4) whether and how the strength of associations changes over the course of development remains largely unclear; (5) also, questions on the reciprocity and causality of the relationship between MC and PA (antecedent/consequent mechanisms) remain unanswered. 
Since the publication of these reviews, a growing number of longitudinal studies investigating the causal or reciprocal relationship between MC and PA, as well as the potential mediators of this relationship in childhood and adolescence, have been noted. ${ }^{16-20}$ The results of the Copenhagen School Children Intervention Study ${ }^{18}$ indicated a reciprocal relationship between MC and PA over 7 years from early to late childhood. The level of MC achieved in late childhood was predicted by PA in early childhood. Conversely, young children's level of MC proved to be a significant predictor of their PA level 7 years later. This relationship was mediated in both directions by HRF. Mediation via PMC was not tested. Vigorous PA (VPA) was directly and indirectly associated with MC, but moderate-to-vigorous PA (MVPA) and MC are only indirectly connected via HRF mediation. ${ }^{18}$ In addition, crosssectional studies provided preliminary support for the reciprocal nature of the relationship between PA and MC, taking into account PMC or HRF. 16,21,22 In a study with older Finnish children, Jaakkola et $\mathrm{al}^{16}$ tested the longitudinal association between MC and MVPA in both directions, including PMC and HRF as mediators. They interpreted their results as almost fully consistent with the Stodden et $\mathrm{al}^{8}$ concept. The data suggest MVPA as a stronger predictor (directly and indirectly) of MC, as opposed to MC predicting MVPA. PMC and HRF mediated the relationship between MVPA and MC in both directions. ${ }^{16}$ Recent longitudinal studies examining only one direction of the PA-MC relationship also supported the theoretical concept. ${ }^{17,19,23}$ It should be noted that the direct or indirect effects identified in these studies are of small to medium size.

Barnett et al $^{12(\mathrm{p} 1684)}$ recommended further investigating the nuances of the PA-MC relationship "to be able to tease out exactly what sorts of activity better contribute to what sort of $\mathrm{MC}$ (and the reverse) at different ages." Several studies have demonstrated that subdomains of MC could be of different relevance. ${ }^{21,22,24}$ Furthermore, longitudinal studies have suggested that the causal relationship between PA and MC becomes empirically evident with the transition from middle childhood to adolescence. ${ }^{17-19}$

It is also recommended to investigate the importance of different PA intensity levels for the development and promotion of MC, fitness, and health. ${ }^{24}$ Longitudinal studies have shown that the relationship between PA and MC becomes empirically evident in late childhood and early adolescence, particularly when objectively measured VPA is considered. ${ }^{17-19}$ Furthermore, studies with children and adolescents found that VPA is more closely connected to healthrelated fitness and health benefits than lower PA intensity levels. ${ }^{25}$

It is noticeable that each of the 2 directions of the hypothesized reciprocal relationship between $\mathrm{PA}$ and $\mathrm{MC}$ has so far only been tested in isolation. Also, limited studies with a focus on the reciprocal nature of the PA-MC relationship in middle to late childhood have considered PMC, VPA, or subdomains of MC. In the present study, these shortcomings were taken into account. The purpose of this study was to investigate (1) the reciprocal relationship between VPA and $\mathrm{MC}$ and (2) the mediating influence of corresponding selfperceptions of MC on the relationship between VPA and MC.

\section{Methods}

\section{Participants}

The study was conducted in 2 similar schools, located in small towns in rural eastern Germany. A convenience sample of 51 fifth graders $(52.9 \%$ boys, $47.1 \%$ girls) aged between 10 and 11 years (t1: $M=10.27, \mathrm{SD}=0.45)$ participated voluntarily in the study. None of them met the following exclusion criteria: regular drug intake, absence of upper/lower extremity, prevalence of chronic or acute diseases that could possibly restrict PA during everyday life.

\section{Measures}

VPA was measured objectively by triaxial accelerometers (ActiGraph wGT3X-BT, ActiGraph, Pensacola, FL). The devices were worn on the wrist of the nondominant hand during waking and sleeping hours for 7 consecutive days (except during water-based activities). Prior work has suggested that children's compliance is higher when accelerometers are worn on the wrist compared with on the hip. ${ }^{26}$ One conclusion of this finding was that the use of ActiGraph accelerometers on the wrist can encourage increased wear time, which may provide a more accurate assessment of PA under everyday conditions. ${ }^{27}$ Chandler et $\mathrm{al}^{28}$ recommended placement on the nondominant wrist because it may improve user compliance with regard to wear time and monitor position and decrease counts during sedentary activity. To determine the nonwear time, the Troiano algorithm ${ }^{29}$ was used. If the wear time was at least $70 \%$ per day, the measurements were considered valid on those days. In the following data analyses, only students with valid measurements for at least 5 days (with $\geq 1$ weekend day and $\geq 3$ weekdays) were included. ${ }^{30}$ An algorithm proposed by Mattocks et $\mathrm{al}^{31}$ and the cut point of 6130 threshold counts per minute were used to calculate VPA per day (in minutes) with the ActiGraph data analysis software ActiLife (ActiGraph).

MC was recorded with the MOBAK-5-6 test instrument for fifth and sixth graders aged between 10 and 12 years. ${ }^{32}$ The MOBAK-5-6 provides an approach for diagnosing and evaluating context-dependent performance dispositions and enables curricularly valid and grade-specific MC measurements in physical education classes. ${ }^{33,34}$ This instrument focuses on the functional mastery of motor demands and achievement of the movement goal by means of general MC. The operationalization of actual MC is based on the consideration of what a child should be able to perform at a certain age level in order to participate actively in the culture of sport and exercise. With 8 items (Table 1), MOBAK5-6 depicts 2 factors that can be understood as MC: self-movement (MC-SM; balancing, rolling, jumping, and running) and object movement (MC-OM; throwing, catching, bouncing, and dribbling). Each test item must be explained by a test leader (including a one-time demonstration of correct performance) immediately before the children perform it. The children had several attempts each (no trial run) to complete the test items. MC-OM and MC$\mathrm{SM}$ are sum scores (with a value range of 0-8) calculated from results on each of the 4 content-related MOBAK-5-6 test items. High scores indicate well-developed MC. A more accurate description of the assessment procedure is provided in Table 1 and the test manual. ${ }^{32}$ Herrmann and Seelig ${ }^{34}$ reported initial evidence for the construct and criteria validity of MOBAK-5-6. This study confirms that the test items represent a 2-factor structure with a satisfactory overall factor reliability (MC-OM: FR $=0.85$; MCSM: FR =0.59).

PMC was measured with the SEMOK (Selbstwahrnehmung der motorischen Kompetenz) questionnaire for fifth-grade students and older. ${ }^{9}$ The 8 items of the SEMOK are designed to complement the 8 items of the MOBAK-5-6 (Table 1). They refer to children's self-assessment of whether they are capable of meeting the basic motor demands formulated from physical education curriculum standards. Equivalent to the 2-factor structure of MOBAK-5-6, SEMOK items also depict 2 factors: self-movement (PMC-SM; based on self-perceptions regarding balancing, rolling, jumping, 


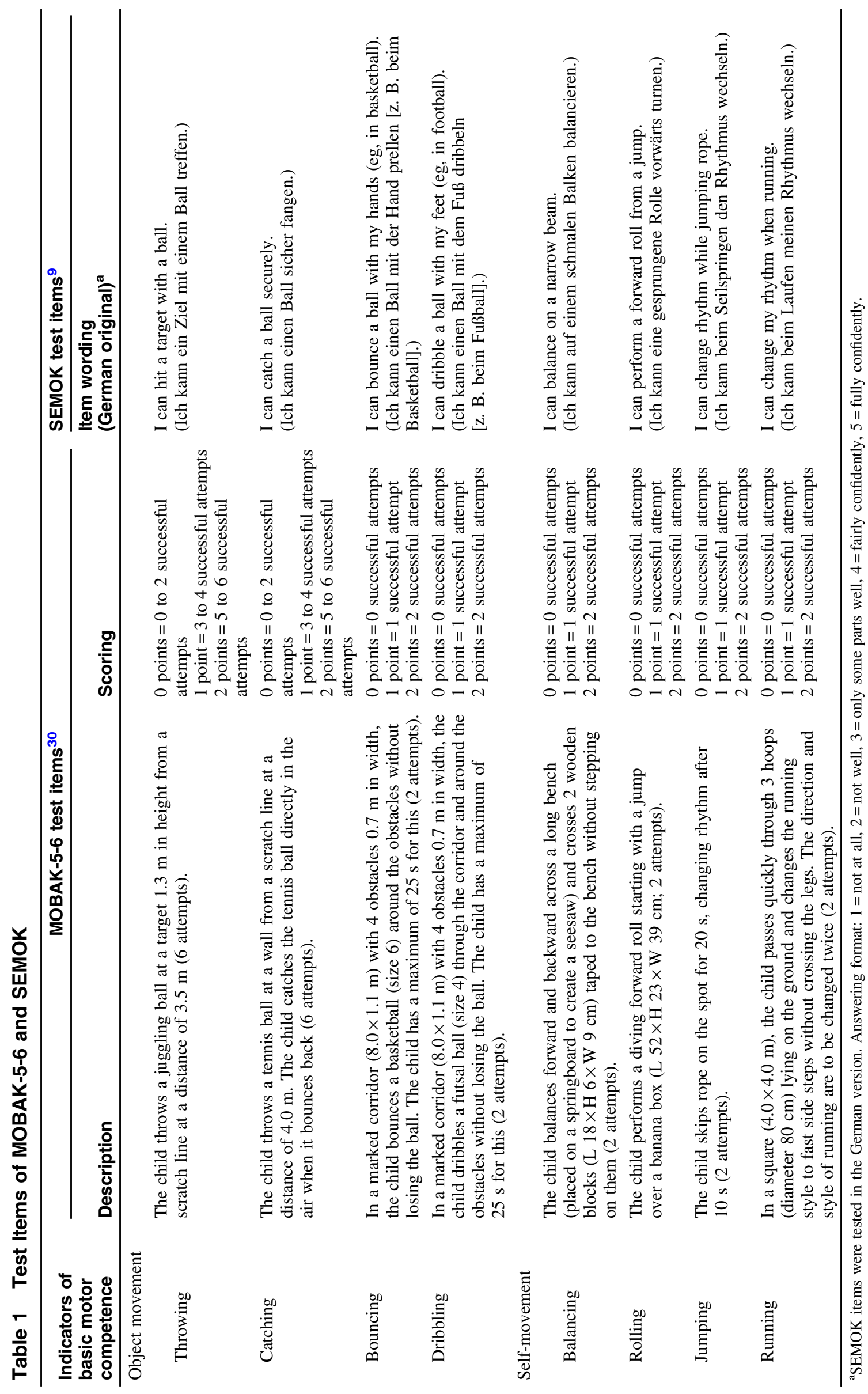


and running) and object movement (PMC-OM; based on selfperceptions regarding throwing, catching, bouncing, and dribbling). PMC-OM and PMC-SM are mean scores (with a value range of 1-5) calculated from self-reported information on each of the 4 content-related SEMOK items. High scores indicate positive self-perceptions of MC. As part of the validation study, ${ }^{9}$ (1) the construct and criteria validity of SEMOK were evaluated successfully, (2) a tight link between MC and PMC was verified, and (3) the 2-factor model showed good overall FR (PMC-OM: $\mathrm{FR}=0.75 ; \mathrm{PMC}-\mathrm{SM}: \mathrm{FR}=0.77$ ).

\section{Procedure}

This investigation was designed as a longitudinal field study with 2 points of measurement 10 months apart (t1: August 2016, t2: June 2017). Data were collected by research assistants. In a 3-hour training session, they were introduced to the test instruments and protocols. The teachers of the tested children were not involved in the data collection. At both times, VPA was measured first. After measuring VPA, MC was tested on the same day in sports halls during physical education lessons. Classes were divided into 5 to 8 small groups of 3 to 4 children. Each group was guided and assessed live by a research assistant. During the test sessions, all research assistants were supervised by a senior researcher who is a professional in sports and educational sciences. One test session lasted between 50 and 70 minutes. One week before $\mathrm{MC}$ was tested at $\mathrm{t} 2$, PMC was recorded within 4 to 6 minutes during a classroom survey. The testing order was chosen to (1) ensure the necessary temporal proximity of the tests and (2) prevent the participants' experiences and results of the MC assessment from influencing their current PMC and everyday PA.

The study was approved by the local school ministries and the University of Leipzig (Medical Faculty) ethics committee. All research procedures and methods in this study were in line with the Declaration of Helsinki. Assent and informed consent were provided by the children and their legal guardians, respectively.

\section{Data Analysis}

Data analyses were performed using Mplus (version 8.4; Muthén \& Muthén, Los Angeles, CA). ${ }^{35}$ The data were thoroughly tested for their suitability for the applied statistical methods. No serious violations of requirements (eg, homoscedasticity, linearity, normality of residuals) were identified. Four saturated pathway models in a cross-lagged panel design were analyzed. These models contained only manifest variables (Figures 1 and 2). Models A1 and $\mathrm{A} 2$ focused on the relationship between VPA and MC-OM. In models B1 and B2, VPA was associated with MC-SM. All models considered the VPA and MC data from t1 to $\mathrm{t} 2$. In addition, the self-perception of $\mathrm{MC}$ at $\mathrm{t} 2$ was included as a mediator variable in models A2 (PMC-OM) and B2 (PMC-SM). Parameters were estimated using the robust maximum likelihood estimation algorithm, and the models in which the variables had missing values were estimated using the full information maximum likelihood algorithm. ${ }^{36}$ The data analysis included 15 students with partially incomplete data. There were 21 missing single values randomly
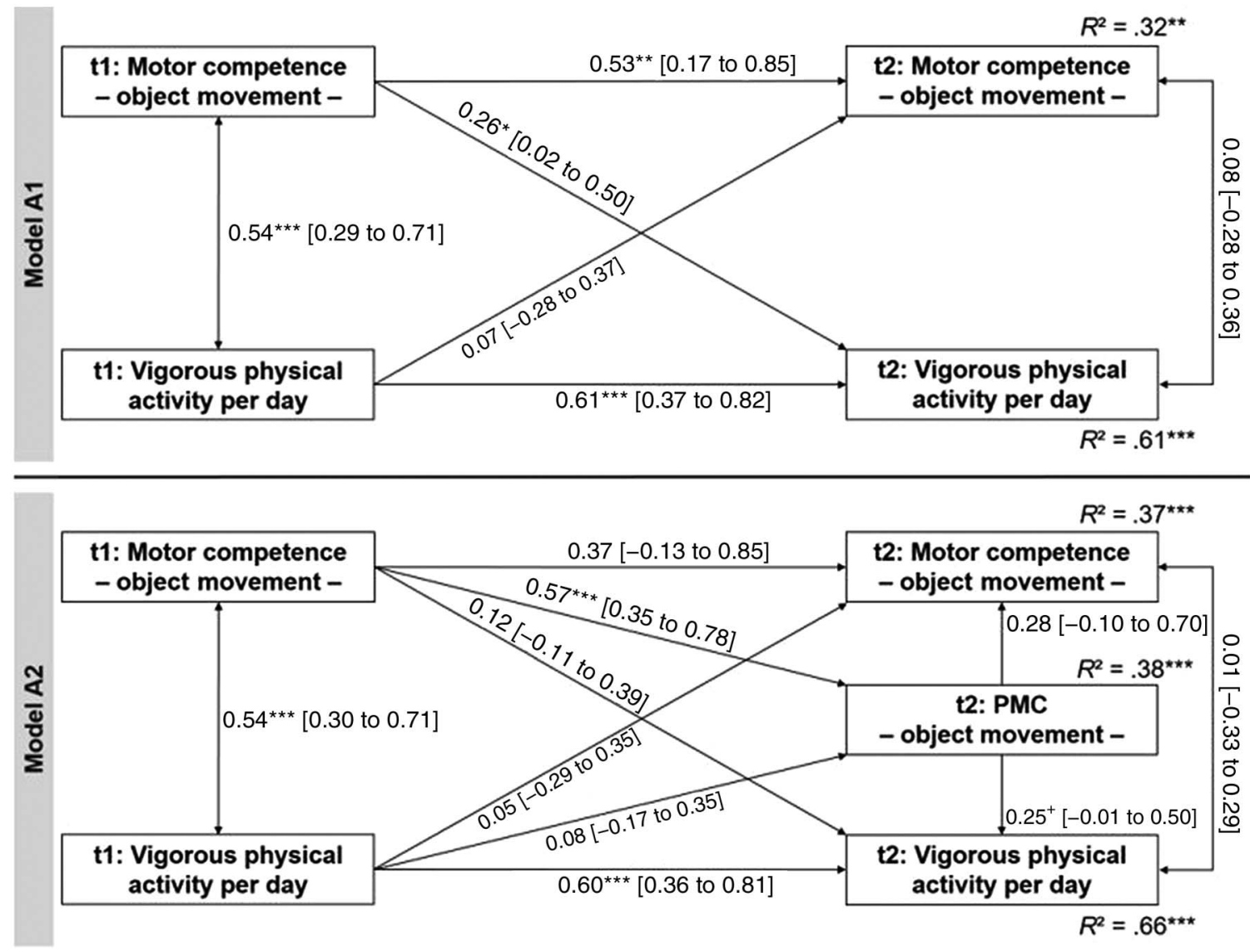

Figure 1 - Pathway models with a focus on the relationships between motor competence (object movement), vigorous physical activity, and PMC (perceived motor competence; object movement). Coefficients of determination $\left(R^{2}\right)$, standardized regression coefficients $(\beta)$, and $95 \%$ confidence intervals are presented. $* P \leq .05, * * P \leq .01, * * * P \leq .001,{ }^{+} P \leq .10$. 
appearing throughout all items $(5.2 \%)$. The main reason was illness-related absence from physical education. Standardized $R^{2}$ and $\beta$ values, corresponding 95\% confidence intervals (CIs; based on 5000 bias-corrected bootstraps), and $P$ values were used to describe total, direct, and indirect effects. Indirect effects (based on mediation via PMC) were tested with a variant of the Sobel test implemented in Mplus. A 95\% CI without including zero was primarily used to identify significant effects but also a $P$ value $\leq .05$. Effect sizes were interpreted as small $\left(R^{2}>.02, r>.10, \beta>0.05\right)$, medium $\left(R^{2}>.15, r>.30, \beta>0.25\right)$, and large $\left(R^{2}>.35, r>.50\right.$, $\beta>.45) .{ }^{37,38}$

\section{Results}

Descriptive statistics are reported in Table 2. Bivariate analyses of relationships among individual data at $\mathrm{t} 1$ and $\mathrm{t} 2$ suggested a high temporal stability of VPA $(r=.76, P<.001)$, MC-OM $(r=.57$, $P<.001)$, and MC-SM $(r=.64, P<.001)$. In the multivariate and longitudinal analyses, the temporal stabilities of VPA and MC from t 1 to 2 were reflected in significant pathways with large effect sizes (Figure 1: model A1; Figure 2: model B1). For example, the majority of children who spent more time on VPA or had a higher level of $\mathrm{MC}$ than other children at $\mathrm{t} 1$ also did so at $\mathrm{t} 2$.

\section{Reciprocal Relationships Between VPA and MC}

There is a strong and positive association between MC-OM and VPA at t1 (Figure 1: model A1). However, a cross-sectional association between MC-SM and VPA is absent at both time points (Figure 2: model B1). With a view of the cross-pathways in models $\mathrm{A} 1$ and $\mathrm{B} 1, \mathrm{VPA}$ at $\mathrm{t} 1$ does not contribute to the explanation of MC-SM or MC-OM at $\mathrm{t} 2$. The variances in MC$\mathrm{OM}$ and MC-SM at $\mathrm{t} 2$ are explained solely by the previous level of the same MC subdomain. For the reverse direction, only the crosspathway from MC-OM at t1 to VPA at 2 was significant. The level of MC-SM at $\mathrm{t} 1$ does not seem to be relevant for later engagement in VPA (Figure 1: model A1; Figure 2: model B1).

Table 2 Results of Descriptive Analysis

\begin{tabular}{lccccc}
\hline Variables & $\begin{array}{c}\text { Number } \\
\text { of items }\end{array}$ & $\begin{array}{c}\text { Measurement } \\
\text { time }\end{array}$ & N & M & SD \\
\hline $\begin{array}{l}\text { VPA (aver- } \\
\text { age }\end{array}$ & 1 & $\mathrm{t} 1$ & 47 & 51.35 & 26.72 \\
$\begin{array}{l}\text { minutes } \\
\text { per day) }\end{array}$ & & $\mathrm{t} 2$ & 45 & 52.74 & 20.38 \\
$\begin{array}{l}\text { MC-OM } \\
\text { sum score }\end{array}$ & 4 & $\mathrm{t} 1$ & 51 & 3.04 & 2.00 \\
of 0-8) & & $\mathrm{t} 2$ & 46 & 4.09 & 1.92 \\
$\begin{array}{l}\text { MC-SM } \\
\text { (sum score }\end{array}$ & 4 & $\mathrm{t} 1$ & 51 & 3.51 & 1.72 \\
$\begin{array}{l}\text { of 0-8) } \\
\text { PMC-OM } \\
\text { (mean score }\end{array}$ & 4 & $\mathrm{t} 2$ & 44 & 4.52 & 1.82 \\
$\begin{array}{l}\text { of 1-5) } \\
\begin{array}{l}\text { PMC-SM } \\
\text { (mean score }\end{array}\end{array}$ & 4 & $\mathrm{t} 2$ & 51 & 3.87 & 0.75 \\
of 1-5) & & & & & \\
\hline
\end{tabular}

Abbreviations: MC, motor competence; OM, object movement; PMC, perceived motor competence; SM, self-movement; VPA, vigorous physical activity.

\section{PMC as a Mediator}

With the inclusion of PMC as a mediator in the reciprocal relationship analysis, the temporal stability of MC-SM and VPA between $\mathrm{t} 1$ and $\mathrm{t} 2$ remained widely unchanged. However, the inclusion of PMC-OM led to a weakening of the direct association between MC-OM at $\mathrm{t} 1$ and MC-OM at $\mathrm{t} 2$. In the 2 models with $\mathrm{PMC}$ as a mediator, the proportion of explained variance $\left(R^{2}\right)$ regarding MC-OM at $\mathrm{t} 2(+5 \%), \mathrm{MC}-\mathrm{SM}$ at $\mathrm{t} 2(+1 \%)$, and VPA at t2 (model A2: 5\%, model B2: $+7 \%$ ), was higher than before. In addition, VPA at $\mathrm{t} 1$ was not related to either PMC-OM or PMC-SM at $\mathrm{t} 2$ (Figures 1 and 2), so that VPA at $\mathrm{t} 1$ also had no specific indirect effects on MC-SM at $22(\beta=0.00 ; P=.920 ; 95 \% \mathrm{CI},-0.06$ to 0.09$)$ and $\mathrm{MC}-\mathrm{OM}$ at $\mathrm{t} 2(\beta=0.02 ; P=.626 ; 95 \% \mathrm{CI},-0.03$ to $0.17)$. Consequently, there are no significant total effects of VPA at t1 on MC-SM at t $2(\beta=0.16 ; P=.251 ; 95 \% \mathrm{CI},-0.12$ to 0.42$)$ and on $\mathrm{MC}-\mathrm{OM}$ at $\mathrm{t} 2(\beta=0.07 ; P=.683 ; 95 \% \mathrm{CI},-0.29$ to 0.37$)$.

The results for model A2 showed a moderate total effect of MC-OM at $\mathrm{t} 1$ on VPA at $\mathrm{t} 2(\beta=0.26 ; P=.025 ; 95 \% \mathrm{CI}, 0.02$ to 0.49 ), but no direct effect as found in model A1 (Figure 1). This relationship seems to be mediated via PMC-OM (indirect effect: $\beta=0.14 ; P=.077 ; 95 \% \mathrm{CI}, 0.01$ to 0.31$)$. A strong, positive relationship was revealed between $\mathrm{MC}-\mathrm{OM}$ at $\mathrm{t} 1$ and $\mathrm{PMC}-\mathrm{OM}$ at $\mathrm{t} 2$, meaning that a higher level of $\mathrm{MC}$ may lead to a more positive self-perception of MC in the future. However, a significant increase in VPA due to a more positive PMC-OM could not be definitely determined, although the effect size was moderate $(\beta=0.25$; $P=.060 ; 95 \% \mathrm{CI},-0.01$ to 0.50$)$. In model A2, there was also an insignificant relationship between PMC-OM and MC-OM at $\mathrm{t} 2$.

Model B2 (Figure 2) indicates neither a total effect $(\beta=0.02$; $P=.823 ; 95 \% \mathrm{CI},-0.19$ to 0.24$)$ nor a direct effect of MC-SM at $\mathrm{t} 1$ on VPA at $\mathrm{t} 2$ but a small indirect effect via PMC-SM $(\beta=0.13$; $P=.023 ; 95 \%$ CI, 0.04 to 0.26 ). Similar to MC-OM in model A2, a higher level of MC-SM at 11 promotes more positive self-perception in the corresponding subdomain of $\mathrm{MC}$ at $\mathrm{t} 2$. Furthermore, a more positive PMC-SM contributes to significantly increased time per day in VPA at $\mathrm{t} 2$ (model B2).

Comparing models A2 and B2, it is remarkable that MC-OM at $\mathrm{t} 1$ explained $38 \%$, and $\mathrm{MC}-\mathrm{SM}$ at $\mathrm{t} 126 \%$, of the variance in the corresponding PMC. However, the proportion of explained variance in the 2 dependent variables (MC and VPA at t2) is of similar magnitude in both models.

\section{Discussion}

The aim of this study was to investigate reciprocal relationships among VPA, MC, and PMC. Pathway models in a cross-lagged panel design were created to examine both directions of the reciprocal relationship between MC and VPA simultaneously, including PMC as a mediator. To our knowledge, this is the first longitudinal study to test assumptions of the theoretical concept of Stodden et $\mathrm{al}^{8}$ in this way.

In accordance with the theoretical concept,${ }^{8}$ it was expected that children from middle childhood onward would be able to progressively assess their MC realistically. This assumption is supported by the revealed large and positive effect of $\mathrm{MC}$ at $\mathrm{t} 1$ on $\mathrm{PMC}$ at $\mathrm{t} 2$. This relationship was also found-albeit less strong-by cross-sectional studies. ${ }^{16,21,22}$ The different effect sizes may be due to a more general assessment of PMC that was not aligned with the assessed MC in these studies. ${ }^{10}$

It was also hypothesized that from the moment children begin to perceive their MC more realistically, the MC-PA relationship 


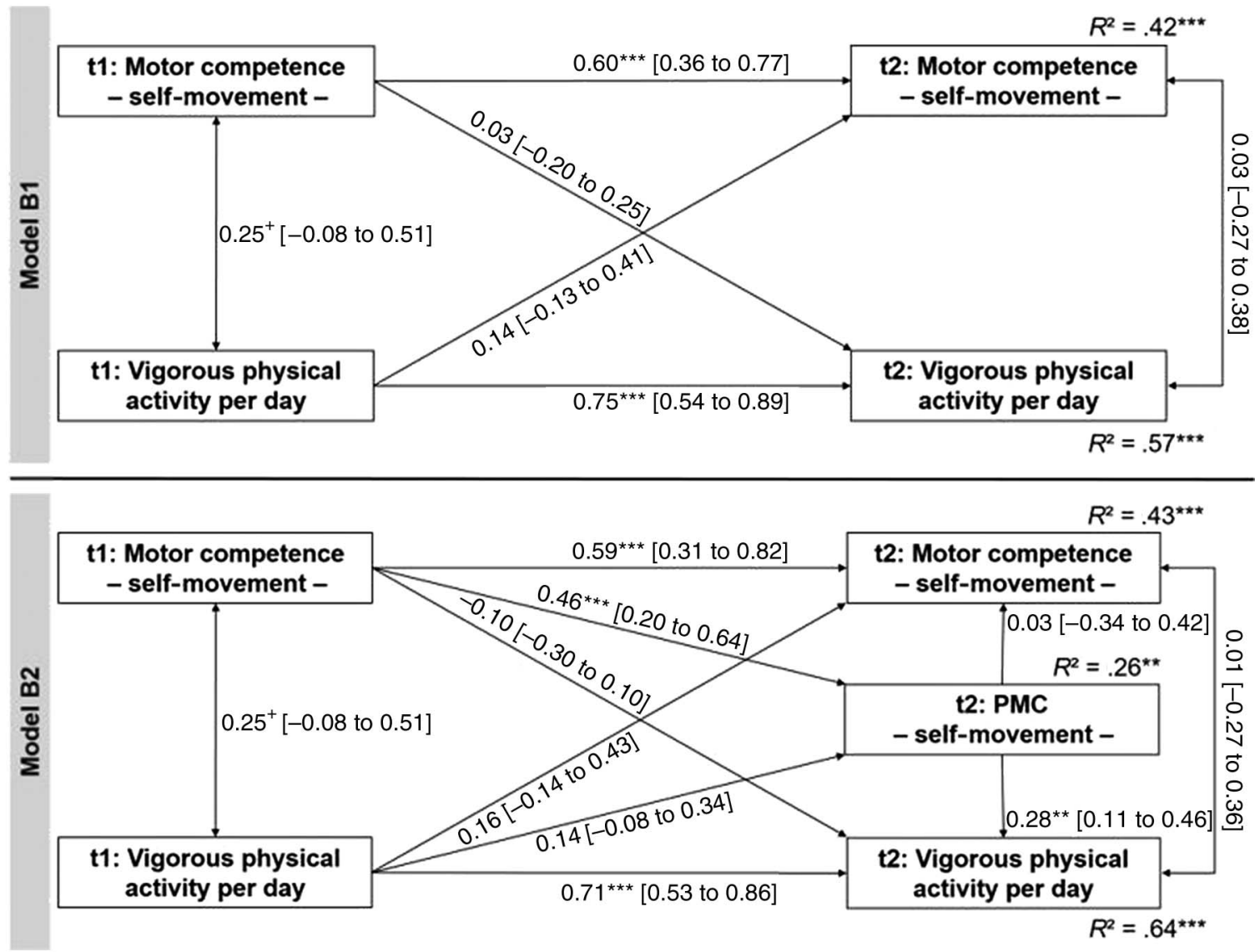

Figure 2 - Pathway models with a focus on the relationships between motor competence (self-movement), vigorous physical activity, and PMC (perceived motor competence; self-movement). Coefficients of determination $\left(R^{2}\right)$, standardized regression coefficients $(\beta)$, and $95 \%$ confidence intervals are presented. $* P \leq .05, * * P \leq .01, * * * P \leq .001,{ }^{+} P \leq .10$.

will be increasingly mediated by PMC. The indirect effect of MC-SM at $\mathrm{t} 1$ on VPA at $\mathrm{t} 2$ revealed here indicates the importance of PMC as a mediator. This result is in accordance with the theory and suggests that higher levels of MC may provide greater opportunities to engage in various and more demanding physical activities. Previous studies also found indirect effects of similar size. ${ }^{16,21,22}$ Contrary to these studies, no direct effect of MC on PA was detected as soon as PMC was considered to be a mediator. An obvious reason for this might be the aforementioned strength of the longitudinal association between MC and PMC. However, the positive and direct relationship between PMC and VPA at t2 corresponds to the theoretical concept ${ }^{8}$ and previous findings. ${ }^{11,16,23}$

Contrary to the assumption of a causal and reciprocal relationship between PA and MC, no direct or indirect effect of VPA at $\mathrm{t} 1$ on $\mathrm{MC}$ at $\mathrm{t} 2$ was found. This result also seems to contradict the results of other studies, which found indirect effects (via PMC) and, occasionally, direct effects of PA on MC. ${ }^{16,21,23}$ This difference may be due to the fact that these studies used cross-sectional data, referred to slightly older participants, and tested PMC not in relation to MC using a task-specific and aligned scale. The last point should especially be emphasized because no evidence for the impact of $\mathrm{PA}$ on $\mathrm{PMC}^{39}$ or $\mathrm{PMC}$ on $\mathrm{MC}^{40}$ was found in studies using aligned instruments for $\mathrm{MC}$ and PMC. Barnett et $\mathrm{al}^{39}$ explained the absence of a pathway from PA to PMC by pointing out that children may not perceive their MC as related to their engagement in (many) different types of PA (eg, swimming, cycling, climbing). To attain a more accurate understanding of the impact of PA on PMC, they suggested assessing self-perception in these types of PA, or specifically assessing MC as relevant to these different types of PA.

In summary, the assumed reciprocal relationship between $\mathrm{MC}$ and PA was not supported by this study's findings. MC seems to drive PA (at least indirectly) but not the reverse. Several studies have found different results for the associations among PA, MC, and PMC depending on the considered subdomains of MC..$^{21,22,24,40}$ The comparison of object movement and self-movement only revealed that the cross-sectional and longitudinal associations between MC-OM and VPA tend to be slightly closer than between MC-SM and VPA when PMC is not considered to be a mediator. On the other hand, the comparison of subdomain-specific models A2 and B2 revealed similarities in the relationships between MC-VPA, MC-PMC, and PMC-VPA. However, the remarkable similarities between the 2 subdomains of MC support the maintenance of nonspecifically formulated assumptions in the theoretical concept. ${ }^{8}$

Besides the aforementioned strengths of this study, several limitations should be considered. First, the given sample allowed us to analyze saturated path models with the necessary test power to identify statistically significant medium to large effects. However, the sample size may not be sufficient to detect even small effects reliably. Therefore, the results should be interpreted with caution. Second, a more differentiated analysis of path models considering the potential impact of sex, body mass index, and socioeconomic status had to be omitted. Scientific reviews pointed out that these personal characteristics can have moderation effects on the 
relationships between MC, PMC, and PA. 7,11,14,15 Third, the analyzed data were partially incomplete. The missing values occurred mainly in the repeated collection of accelerometer data. A clearing of missing values using the full information maximum likelihood estimation algorithm was required. Although this may have caused data bias, there is some evidence that accelerometer data were considered to be missing at random..$^{41}$ Fourth, this study did not include HRF as a potential mediator besides PMC, which could provide more insights into the relationship between PA and MC. ${ }^{8}$ Fifth, due to the selected study period of 10 months and the age-homogeneous sample, only limited insights into the development of MC, PMC, VPA, and their relationships are given. Sixth, the consideration of MC and PMC is limited to object movement and self-movement. Other aspects of basic MC (eg, object locomotion, moving in water), which are also relevant for the promotion of motor development in school-based physical education, were not taken into account. It could be beneficial to use a more comprehensive measurement of MC and PMC to clarify the research questions examined here. ${ }^{42}$ Last but not least, it should be noted that, with VPA, only a small part of children's PA was considered here. The results may not be generalizable to total PA or lower PA intensity levels. Previous studies have shown that the relationship between PA and MC are not independent of the PA intensity. ${ }^{18,19}$

\section{Conclusion}

VPA did not predict MC. Conversely, MC-OM and MC-SM proved to be predictive of VPA after 10 months, but only when the relationship was mediated by the corresponding PMC. The comparison of object movement and self-movement revealed more similarities than differences in the examined relationships. The results correspond partially with the theoretical concept ${ }^{8}$ and previous findings. This study suggests that $\mathrm{MC}$ and PMC should be considered as basic components in promoting children's VPA. Further longitudinal studies with larger samples, including different PA intensity levels and more moderator variables, are required to confirm, refute, or further deepen the presented results.

\section{References}

1. Janssen I, LeBlanc AG. Systematic review of the health benefits of physical activity and fitness in school-aged children and youth. Int $J$ Behav Nutr Phys Act. 2010;7(40):1-16. doi:10.1186/1479-5868-7-40

2. Kohl HW, Craig CL, Lambert EV, et al. The pandemic of physical inactivity: global action for public health. Lancet. 2012;380(9838):294 305. PubMed ID: 22818941 doi:10.1016/S0140-6736(12)60898-8

3. European Commission, Expert Group on Health-enhancing Physical Activity. Recommendations to encourage physical education in schools, including motor skills in early childhood, and to create valuable interactions with the sport sector, local authorities and the private sector. 2015. http://ec.europa.eu/transparency/regexpert/ index.cfm?do=groupDetail.groupDetailDoc\&id=19860\&no=12015. Accessed July 16, 2019.

4. World Health Organization. Physical Activity Strategy for the WHO European Region 2016-2025. Copenhagen, Denmark: World Health Organization; 2016.

5. Heath GW, Parra DC, Sarmiento OL, et al. Evidence-based intervention in physical activity: lessons from around the world. Lancet. 2012;380(9838):272-281. PubMed ID: 22818939 doi:10.1016/ S0140-6736(12)60816-2
6. Gallahue D, Ozmun J. Understanding Motor Development: Infants, Children, Adolescents, Adults. 6th ed. Boston, MA: McGraw-Hill; 2006.

7. Robinson LE, Stodden DF, Barnett LM, et al. (2015). Motor competence and its effect on positive developmental trajectories of health. Sports Med. 2015;45(9):1273-1284. PubMed ID: 26201678 doi:10. 1007/s40279-015-0351-6

8. Stodden DF, Goodway JD, Langendorfer SJ, et al. A developmental perspective on the role of motor skill competence in physical activity: an emergent relationship. Quest. 2008;60(2):290-306. doi:10.1080/ 00336297.2008.10483582

9. Herrmann C, Seelig H. "I can dribble!" On the relationship between children's motor competencies and corresponding self-perceptions. Ger J Exerc Sport Res. 2017;47(4):324-334. doi:10.1007/s12662017-0468-x

10. Estevan I, Barnett LM. Considerations related to the definition, measurement and analysis of perceived motor competence. Sports Med. 2018;48(12):2685-2694. PubMed ID: 29938348 doi:10.1007/ s40279-018-0940-2.

11. Babic MJ, Morgan PJ, Plotnikoff RC, Lonsdale C, White RL, Lubans DR. Physical activity and physical self-concept in youth: systematic review and meta-analysis. Sports Med. 2014;44(11):1589-1601. PubMed ID: 25053012 doi:10.1007/s40279-014-0229-Z

12. Barnett LM, Lai SK, Veldman SLC, et al. Correlates of gross motor competence in children and adolescents: a systematic review and meta-analysis. Sports Med. 2016;46(11):663-688. doi:10.1007/ s40279-014-0229-z

13. Cattuzzo MT, Henrique RS, Ré AHN, et al. Motor competence and health related physical fitness in youth: a systematic review. J Sci Med Sport. 2016;19(2):123-129. PubMed ID: 25554655 doi:10.1016/ j.jsams.2014.12.004

14. Holfelder B, Schott N. Relationship of fundamental movement skills and physical activity in children and adolescents: a systematic review. Psychol Sport Exerc. 2014;15(4):382-391. doi:10.1016/j.psychsport. 2014.03.005

15. Logan SW, Webster EK, Getchell N, Pfeiffer KA, Robinson LE. Relationship between fundamental motor skill competence and physical activity during childhood and adolescence. A systematic review. Kinesiol Rev. 2015;4(4):416-426. doi:10.1123/kr.2013-0012

16. Jaakkola T, Huhtiniemi M, Salin K, Seppälä S, Lahti J, Hakonen H. Motor competence, perceived physical competence, physical fitness, and physical activity within Finnish children. Scand J Med Sci Sports. 2019;29(7):1013-1021. PubMed ID: 30825382 doi:10.1111/sms.13412

17. Jaakkola T, Yli-Piipari S, Huotari P, Watt A, Liukkonen J. Fundamental movement skills and physical fitness as predictors of physical activity: a 6-year follow-up study. Scand J Med Sci Sports. 2016; 26(1):74-81. PubMed ID: 25644386 doi:10.1111/sms.12407

18. Lima RA, Pfeifer K, Larsen LR, et al. Physical activity and motor competence present a positive reciprocal longitudinal relationship across childhood and early adolescence. J Phys Act Health. 2017;14(6):440447. PubMed ID: 28169569 doi:10.1123/jpah.2016-0473

19. Lopes L, Mota APS, Moreira C, et al. Longitudinal associations between motor competence and different physical activity intensities: LabMed physical activity study. J Sports Sci. 2019;37(3):285-290. PubMed ID: 29996715 doi:10.1080/02640414.2018.1497424

20. McIntyre F, Parker H, Chivers P, Hands B. Actual competence, rather than perceived competence, is a better predictor of physical activity in children aged 6-9 years. J Sports Sci. 2018;36(13):1433-1440. PubMed ID: 29016237 doi:10.1080/02640414.2017.1390243

21. Barnett LM, Morgan PJ, van Beurden E, Ball K, Lubans DR. A reverse pathway? Actual and perceived skill proficiency and physical activity. Med Sci Sports Exerc. 2011;43(5):898-904. PubMed ID: 20962694 doi:10.1249/MSS.0b013e3181fdfadd 
22. Khodaverdi Z, Bahram A, Stodden D, Kazemnejad A. The relationship between actual motor competence and physical activity in children: mediating roles of perceived motor competence and healthrelated physical fitness. J Sports Sci. 2016;34(16):1523-1529. PubMed ID: 26691581 doi:10.1080/02640414.2015.1122202

23. Burns RD, Fu Y. Testing the motor competence and health-related variable conceptual model: a path analysis. J Funct Morphol Kinesiol. 2018;3(61):1-10. doi:10.3390/jfmk3040061

24. Chan CHS, Ha ASC, Ng JYY, Lubans DR. Associations between fundamental movement skill competence, physical activity and psycho-social determinants in Hong Kong Chinese children. J Sports Sci. 2019;37(2):229-236. PubMed ID: 30064306 doi:10.1080/02640414 .2018.1490055

25. Khodaverdi Z, Goodway JD, Stodden DF. Associations between physical activity and health-related fitness: differences across childhood. Turkish J Sport Exerc. 2017;19(2):169-176. doi:10.15314/tsed. 315806

26. Fairclough SJ, Nooan R, Rowlands AV, Van Hees V, Knowles Z, Boddy LM. Wear compliance and activity in children wearing wrist- and hip-mounted accelerometers. Med Sci Sports Exerc. 2016;48(2):245-253. PubMed ID: 26375253 doi:10.1249/MSS. 0000000000000771

27. McLellan G, Arthur R, Buchan DS. Wear compliance, sedentary behaviour and activity in free-living children from hip- and wrist-mounted ActiGraph GT3X+ accelerometers. J Sports Sci. 2018;36(21): 2424-2430. PubMed ID: 29620970 doi:10.1080/02640414.2018 .1461322

28. Chandler JL, Brazendale K, Beets MW, Mealing BA. Classification of physical activity intensities using a wrist-worn accelerometer in 8-12-year-old children. Pediatr Obes. 2016;11(2):120-127. PubMed ID: 25893950 doi:10.1111/ijpo.12033

29. Troiano RP, Berrigan D, Dodd KW, Mâsse LC, Tilert T, McDowell M. Physical activity in the United States measured by accelerometer. Med Sci Sports Exerc. 2008;40(1):181-188. PubMed ID: 18091006 doi:10.1249/mss.0b013e31815a51b3

30. Smith M, Taylor S, Iusitini L, et al. Accelerometer data treatment for adolescents: fitting a piece of the puzzle. Prev Med Rep. 2017;5(1): 228-231. PubMed ID: 28101444 doi:10.1016/j.pmedr.2016.12.010
31. Mattocks C, Leary $S$, Ness A, et al. Calibration of an accelerometer during free-living activities in children. Int J Pediatr Obes. 2007;2(4):218-226. PubMed ID: 17852552 doi:10.1080/17477160701408809 .

32. Herrmann C, Seelig H. MOBAK-5-6: Basic Motor Competencies in Fifth and Sixth Grade. Test Manual. 2nd ed; 2018. http://mobak.info/wpcontent/uploads/2018/06/MOBAK_5-6_engl.pdf. Accessed July 6, 2019.

33. Herrmann C, Bund A, Gerlach E, et al. A review of the assessment of basic motor qualifications and competencies in school. Int J Phys Educ. 2015;52(3):2-13.

34. Herrmann C, Seelig H. Basic motor competencies of fifth graders. Construct validity of the MOBAK-5 test instrument and determinants. Ger J Exerc Sport Res. 2017;47(4):110-121. doi:10.1007/ s12662-016-0430-3

35. Muthén LK, Muthén BO. Mplus User's Guide: Statistical Analysis With Latent Variables. Los Angeles, CA: Muthén \& Muthén; 2012.

36. Arbuckle JL. Full information estimation in the presence of incomplete data. In Marcoulides GA, Schumacker RE, eds. Advanced Structural Equation Modelling. Mahwah, NJ: Erlbaum; 1996:243-277.

37. Cohen J. Statistical Power Analysis for the Behavioral Sciences. 2nd ed. Hillsdale, NJ: Erlbaum; 1988.

38. Peterson RA, Brown SP. On the use of beta coefficients in metaanalysis. J Appl Psychol. 2005;90(1), 175-181. PubMed ID: 15641898 doi:10.1037/0021-9010.90.1.175

39. Barnett LM, Timperio A, Salmon J, Ridgers ND, Lubans DR. What is the contribution of actual motor skill, fitness, and physical activity to children's self-perception of motor competence? J Mot Learn Dev. 2018;6(2):461-473. doi:10.1123/jmld.2016-0076

40. Bolger LE, Bolger LA, O'Neill C, et al. Accuracy of children's perceived skill competence and its association with physical activity. J Phys Activ Health. 2019;16(1):29-36. doi:10.1123/jpah.2017-0371

41. Saint-Maurice PF, Kim Y, Welk GJ. Evidence for data missing at random in youth physical activity monitoring research. J Sports Sci. 2016;35(5):484-490. PubMed ID: 27071002 doi:10.1080/02640414. 2016.1173719

42. Scheuer C, Bund A, Herrmann C. Diagnosis and monitoring of basic motor competencies among third-graders in Luxembourg. An assessment tool for teachers. Meas Phys Educ Exerc Sci. 2019;23(3): 258-271. doi:10.1080/1091367X.2019.1613998 